\title{
Variable Expression
}

National Cancer Institute ( $\mathrm{NCl})$

\section{Source}

National Cancer Institute (NCI). Variable Expression.

Variation in the manner in which a trait is manifested. When there is variable expressivity, the trait may vary in clinical expression from mild to severe. For example, the condition neurofibromatosis type 1 may be mild, presenting with café-au-lait spots only, or may be severe, presenting with neurofibromas and brain tumors. 